Artur Chodkowski ${ }^{1}$, Katarzyna Nabrdalik ${ }^{1}$, Hanna Kwiendacz ${ }^{1}$, Andrzej Tomasik ${ }^{2}$, Wojciech Bartman ${ }^{3}$, Janusz Gumprecht ${ }^{1}$

${ }^{1}$ Department of Internal Medicine, Diabetology and Nephrology in Zabrze, School of Medicine with the Division of Dentistry in Zabrze, Medical University of Silesia, Katowice, Poland

2Second Department of Cardiology in Zabrze, School of Medicine with the Division of Dentistry in Zabrze

Medical University of Silesia, Katowice, Poland

${ }^{3}$ Department of Neurology in Zabrze, School of Medicine with the Division of Dentistry in Zabrze, Medical University of Silesia, Katowice, Poland

\title{
Pentraxin 3 and retinopathy among type 2 diabetic patients in relation to carotid atherosclerosis and systolic and diastolic cardiac function - a pilot study
}

\section{ABSTRACT}

Introduction. Diabetic retinopathy (DR) is the leading cause of vision loss worldwide. Global prevalence of any diabetic retinopathy is assessed to be $35.4 \%$. Several studies proved that chronic low-grade inflammation may be involved in the pathogenesis of DR. Some studies indicate that macroangiopathic diabetic complications may be associated with microangiopathic ones that is why a "common soil" mechanism of diabetic micro- and macroangiogpathy has been proposed. The aim of this study was to evaluate the association of pentraxin 3 (PTX3), an inflammation's biomarker, with diabetic retinopathy in relation to atherosclerosis in carotid arteries and systolic and diastolic cardiac function.

Material and methods. 43 eligible patients with type 2 diabetes were enrolled into the study and dived into two groups on the basis of presence or lack of retinopathy. Anthropometric, biochemical and carotid as well as cardiac ultrasound parameters were analyzed.

Address for correspondence: dr n. med. Katarzyna Nabrdalik

Katedra i Klinika Chorób Wewnętrznych, Diabetologii i Nefrologii, Śląski Uniwersytet Medyczny

Wydział Lekarski z Oddziałem Lekarsko-Dentystycznym

ul. 3 Maja 13-15, 41-800 Zabrze

Phone: +48323704415

e-mail: knabrdalik@yahoo.com

Clinical Diabetology 2018, 7, 4, 196-202

DOI: $10.5603 /$ DK.2018.0016

Received: 18.08.2018 Accepted: 22.08.2018
Results. There was no direct association between PTX3 concentration and the presence of diabetic retinopathy, but there was a significant correlation between PTX3 and $\mathrm{HbA}_{1 \mathrm{c}}$ value, age and IMT (intima media thickness) in carotid arteries among patients with diabetic retinopathy.

Conclusions. There is a great need for further, larger, studies on inflammatory biomarkers such as PTX3 and micro and macrovascular complications of diabetes mellitus in order to detect predisposed patients early enough to implement early therapeutic intervention of this complication. (Clin Diabetol 2018; 7, 4: 196-202)

Key words: diabetes type 2, diabetic retinopathy, pentraxin 3

\section{Introduction}

Type 2 diabetes mellitus (T2DM) is a major concern for health care systems because of its high prevalence and micro- and macrovascular complications that might cause disability and premature death [1-3]. Over the last two decades new therapeutic options such as analogue insulin, GLP-1 agonists, DPP-4 inhibitors, and SGLT-2 inhibitors were widely implemented to clinical practice nevertheless about half of the patients still doesn't reach the target $\mathrm{HbA}_{1 \mathrm{c}}$ value and therefore they are prone to diabetic complications development [3].

One of the microangiopathic complications is diabetic retinopathy which is the most common, avoidable cause of blindness all over the world [4]. It is estimated 
that in the United States of America $40 \%$ of patients with T2DM have diabetic retinopathy and epidemiological data from other countries are similar [5]. The pathogenesis of diabetic retinopathy, as for most diabetic complications, is complex [6]. Since DeFronzo's concept of ominous octet [7] some new observations were made and chronic low-grade inflammation and therefore the activation of the immune system is acknowledged to take part in type 2 diabetes mellitus [8]. The local inflammation, which contributes to endothelial dysfunction is also, next to hyperglicaemia, associated with micro and macroangiopathy. Initially, the progression of diabetic retinopathy is symptomless and gradual but early stages of the disease are mild and can be detected only while screening [9]. One in two patients with T2DM remains undiagnosed [10] and those patients can already develop advanced stages of the diseases' complication. It is also not unusual that patients with already established diabetes mellitus have the diabetic complications diagnosed late, at advanced stages. Due to T2DM high prevalence, there is a need to design studies to find the ways of an early detection of the disease and its complications and to find the solution how to determine groups of patients who are more susceptible to a complication. Biomarkers are thought to have such a potential meaning and therefore there are studies undergoing to determine specific markers for micro and macrovascular complications. Many biomarkers used in cardiovascular risk scoring are well described and standardized, these include: troponins, C-reactive protein (CRP) and some lipids [11, 12] but biomarkers of microvascular complications are lacking. It is proposed, that one of these cardiovascular biomarkers could be pentraxin 3 (PTX3) [13]. Pentraxins, like CRP, are acute phase reactants and one of the major components of non-specific immune response [14, 15]. Pentraxin 3 binds to C1q complement and eliminates immunological complexes via classical activation of its path [16]. It is also a receptor for patogen associated molecular patterns (PAMPs), which are released by cytokines and take part in different phases of inflammatory response, i.e. pathogen and own damaged cells' recognition [17]. PTX3 is also known as TSG-14 [tumor necrosis factor (TNF)-stimulated gene 14] or TNFAIP-5 (tumor necrosis factor alpha-induced protein 5) and is produced mainly by endothelium, fibroblasts, dendritic cells, macrophages, monocytes, adipocytes, synoviocytes, chondrocytes, renal epithelium and smooth muscle cells. Its production is initiated by mediators such as interleukin $1 \beta($ IL1 $\beta)$, tumor necrosis factor alfa (TNF $\alpha$ ), lipopolisacharydes (LPS), and oxy-LDL lipoproteins [18]. Pentraxin 3 takes part in evolution of inflammation, which is, according to current state of knowledge, one of the factors involved in the pathogenesis of atherosclerosis. Some studies indicate that macroangiopathic diabetic complications may be associated with microangiopathic ones that is why a "common soil" mechanism od diabetic micro- and macroangiogpathy has been proposed and this is the reason why we decided to assess carotid atherosclerosis as well as cardiac systolic and diastolic function among patients with diabetic retinopathy. Diabetic retinopathy on the other hand is one of the microvascular complications, where the role of PTX3 is still under examination but there are some limited reports of its potential association with the presence and severity of retinopathy in patients with diabetes mellitus $[19,20]$.

We have previously examined the association of plasma PTX3 concentration with atherosclerosis in carotid arteries, systolic and diastolic left ventricle function and diabetes control among type 2 diabetic patients in relation to patients without glucose metabolism disorders [21]. This research is a follow up study where we evaluated biochemical, carotid and cardiac ultrasound parameters in the group of diabetic patients dived on the basis of the presence of diabetic retinopathy.

\section{Material and methods}

43 consecutive, eligible T2DM Caucasian patients visiting the Outpatient Diabetology Clinic in Zabrze were enrolled into the study and divided into two groups on the basis of the presence of retinopathy. Clinical and anthropometrical data were collected from patients' medical documentation, among others a fundus examination performed up to 3 months before the enrolment into the study, presence of peripheral neuropathy, and nephropathy. Diabetic nephropathy has been defined as a chronic (lasting more than 3 months) persistence of elevated UACR (Urinary Albumin to Creatinine ratio) and/or over proteinuria and/or decreased below $60 \mathrm{ml} / \mathrm{min} 1.73 \mathrm{~m}^{2}$ renal function assessed with the use of CKD-EPI equation with the lack of signs of other cause of kidney disease. All of the participants gave informed written consent. The inclusion criteria for the study group I were type 2 diabetes mellitus diagnosis and the presence of retinopathy whereas for the group II - type 2 diabetes mellitus and lack of retinopathy. All subjects with end stage renal disease, heart failure (NYHA III and IV), hepatic cirrhosis, anemia and presence of any vascular events in the past 6 months prior to the study (i.e. acute coronary syndrome, percutaneous coronary interventions and stroke) were excluded from the study. All of the patients underwent a carotid ultrasound examination and transthoracic echocardiography. Plasma pentraxin 3 concentration, 
Table 1. Basic clinical and demographic characteristics of the study groups

\begin{tabular}{|c|c|c|c|}
\hline Parameter & Group I & Group II & Statistical analysis \\
\hline Participants, $\mathrm{n}=100 \%$ & $11(25.58 \%)$ & $32(74.42 \%)$ & - \\
\hline Female, n; \% & $3 ; 27.27 \%$ & $17 ; 53.13 \%$ & Pearson $\chi^{2}=2.27 ; p=0.87$ \\
\hline Male, n; \% & $8 ; 72.73 \%$ & $15 ; 46.87 \%$ & \\
\hline Age (years) & $62.63 \pm 4.97$ & $62.25 \pm 9.85$ & $p=0.64$ \\
\hline Body mass index $\left[\mathrm{kg} / \mathrm{m}^{2}\right]$ & $25.76 \pm 2.89$ & $27.38 \pm 3.00$ & $p=0.05$ \\
\hline T2DM duration (years) & $11.18 \pm 2.48$ & $5.78 \pm 3.62$ & $p=0.001$ \\
\hline T2DM duration $>10$ years & $90.91 \%(n=10)$ & $28.13 \%(n=9)$ & Pearson $\chi^{2}=14.3 ; p=0.001$ \\
\hline Arterial hypertension & $81.81 \%(n=9)$ & $43.75 \%(n=14)$ & Pearson $\chi^{2}=5.11 ; p=0.02$ \\
\hline Hypertension duration (years) & $10.64 \pm 2.87$ & $6.69 \pm 3.61$ & $p=0.001$ \\
\hline Acute coronary syndrome & $63.64 \%(n=7)$ & $6.25 \%(n=2)$ & $p=0.001$ \\
\hline Coronary artery bypass grafting & $27.27 \%(n=3)$ & $3.13 \%(n=1)$ & $p=0.44$ \\
\hline Stroke & $36.36 \%(n=4)$ & $0 \%(n=0)$ & $p=0.88$ \\
\hline $\begin{array}{l}\text { Presence of atherosclerotic plaque in ultrasound } \\
\text { carotid examination }\end{array}$ & $45.45 \%(n=5)$ & $25 \%(n=8)$ & $p=0.57$ \\
\hline Diabetic neuropathy & $90.91 \%(n=10)$ & $6.25 \%(n=2)$ & $p=0.03$ \\
\hline Nephropathy & $90.91 \%(n=10)$ & $0 \%(n=0)$ & $p=0.88$ \\
\hline Macrovascular complications & $90.91 \%(n=10)$ & $34.37 \%(n=11)$ & $p=0.01$ \\
\hline Plasma PTX3 concentration $[\mathrm{ng} / \mathrm{ml}]$ & $1.01 \pm 0.87$ & $1.31 \pm 0.64$ & $p=0.054$ \\
\hline Albuminuria $[\mathrm{mg} / \mathrm{g}]$ & $20.21 \pm 13.61$ & $29.62 \pm 11.24$ & $p=0.05$ \\
\hline eGFR $\left[\mathrm{ml} / \mathrm{min} / 1.73 \mathrm{~m}^{2}\right]$ & $83.5 \pm 20.68$ & $71.56 \pm 19.10$ & $p=0.06$ \\
\hline $\mathrm{HbA}_{1 \mathrm{c}}(\%)$ & $6.02 \pm 0.75$ & $6.4 \pm 0.92$ & $p=0.21$ \\
\hline
\end{tabular}

All the values are presented as mean \pm standard deviation or the percentage of participants

Group I - patients with retinopathy; Group II — patients without retinopathy; T2DM — diabetes mellitus type 2; PTX3 - pentraxin 3; eGFR — estimated glomerular filtration rate; $\mathrm{HbA}_{1 \mathrm{c}}$ - glycated haemoglobin

haemoglobin $A_{1 c}$ value, cholesterol and triglycerides, serum creatinine and urinary albumin secretion were measured. More detailed description of the study and laboratory examination methods were described previously [21]. All statistical analysis were performed using STATISTICA 12.5 (StatSoft, Poland). The qualitative traits were presented as absolute numbers or percentages. The following tests were used: the $\chi^{2}$ test, t-Student test, Mann-Whitney U-test, Kruskal-Wallis test when appropriate. In order to rang correlations Spearman's rank correlation coefficient was used. A $p$ value less than 0.05 was considered significant.

The study was conducted in accordance to the Helsinki Declaration and was approved by the Ethical Committee of Medical University of Silesia.

\section{Results}

Basic demographic and clinical characteristics of the study population are presented in Table 1. In comparison with patients from group II (without retinopathy), patients classified as group I (with retinopathy) had a significantly longer diabetes mellitus (arbitrarily determined as 10 years of the disease) and hypertension duration. In more than $90 \%$ of patients with retinopathy other complications of diabetes oc- curred that is macrovascular complications, diabetic neuropathy and nephropathy. In the group of patients with retinopathy there was not significantly lower plasma PTX3 concentration (Table 1; $\mathrm{p}=0.054$ ). Moreover, in this group there was a lower $\mathrm{HbA}_{1 \mathrm{c}}$ value ( $p=0.21$ ), worse blood flow and intima-media thickness parameters in carotid arteries (CCA IMT av $p=$ 0.13; CCA IMT $\max p=0.33$; ICA MV $p=0.24$; ICA PI $p=0.8$; ICA RI $p=0.51-$ all the abbreviations explained in Table 2), but no significance was found. As for significant differences, in the group I (with retinopathy) patients had lower left ventricle systolic function ( $E F$, ejection fraction) $(p=0.014)$ and worse left ventricle's diastolic function in tissue Doppler imaging $\left(E^{\prime} / A^{\prime}\right)$ with the level of significance $p=0.001$ (summarized in Table 2).

There was a significant correlation between plasma PTX3 concentration and patient's age in both groups (Table $3 ; p=0.005$ ). We also found a high correlation for PTX3 concentration and $\mathrm{HbA}_{1 \mathrm{c}}$ value in a group of patients with retinopathy $(p=0.004)$. Besides, PTX3 concentration was associated with the duration of hypertension in this group of patients $(p=0.001)$.

There was no association of PTX3 concentration with presence of atherosclerotic plaques in carotid 
Table 2. Carotid arteries ultrasound examination and echocardiographic examination results

\begin{tabular}{lccc}
\hline Parameter & Group I & Group II & Statistical analysis \\
\hline CCA IMT av [mm] & $0.81 \pm 0.24$ & $0.67 \pm 0.11$ & $\mathrm{p}=0.13$ \\
CCA IMT max [mm] & $1.10 \pm 0.26$ & $0.98 \pm 0.16$ & $\mathrm{p}=0.33$ \\
ICA MV [cm/s] & $35.33 \pm 10.2$ & $30.54 \pm 7.3$ & $\mathrm{p}=0.24$ \\
ICA PI (pulsatility index) & $1.05 \pm 0.21$ & $1.04 \pm 0.34$ & $\mathrm{p}=0.80$ \\
ICA RI (resistance index) & $0.67 \pm 0.10$ & $0.72 \pm 0.32$ & $\mathrm{p}=0.51$ \\
EF (\%) & $47.7 \pm 12.9$ & $55.84 \pm 5.9$ & $\mathrm{p}=0.014$ \\
E/A & $0.99 \pm 0.25$ & $1.08 \pm 0.20$ & $\mathrm{p}=0.63$ \\
E'/A' $^{\prime}$ & $0.93 \pm 0.2$ & $1.06 \pm 0.18$ & $\mathrm{p}=0.001$ \\
\hline
\end{tabular}

All the values are presented as mean \pm standard deviation

CCA (IMT) av - common carotid artery (intima-media thickness) average; CCA (IMT) max - common carotid artery (intima-media thickness) maximum; ICA (MV) - internal carotid artery (mean velocity); ICA (PI) - internal carotid artery (pulsatility index); ICA (RI) — internal carotid artery (resistance index); $\mathrm{EF}$ - ejection fraction; E/A - E/A ratio in conventional Doppler echocardiography

Table 3. The correlation between PTX3 concentration with given parameters - presented as R Spearman correlation

\begin{tabular}{lcc}
\hline & Group I & Group II \\
\hline Age & $0.61^{*}$ & $0.33^{*}$ \\
$\mathrm{HbA}_{1 \mathrm{c}}$ & $0.53^{*}$ & 0.18 \\
Body mass index & -0.44 & -0.08 \\
eGFR & -0.13 & -0.24 \\
T2DM duration & 0.33 & -0.19 \\
Hypertension duration & $0.58^{*}$ & 0.08 \\
\hline
\end{tabular}

${ }^{*} \mathrm{p}<$ 0.05; eGFR - estimated glomerular filtration rate

arteries in none of the groups (Table 4) nor systolic or diastolic left ventricle function in both groups (Table 4) found. Nevertheless, in the group of patients with retinopathy, plasma PTX3 concentration was significantly correlated with the maximal $(p=0.002)$ and mean ( $p=0.001$ ) intima-media thickness in the common carotid artery (Table 5).

\section{Discussion}

Diabetic complications may be present at the onset of the diabetes however a risk of their occurrence increases over time [22]. Most of patients in presented study had a history of T2DM lasting more than 10 years, so it may be assumed that they were more prone to diabetic complication. The most common diabetic complications were neuropathy, nephropathy and macrovascular complications. About $90 \%$ of patients with diabetic retinopathy had also macrovascular complications what is consistent with the "common soil" theory of vascular complications in diabetes, which was also confirmed by Bartman et al., who described

Table 4. The comparison of PTX3 concentration depending on the presence of atherosclerosis in carotid arteries and cardiac function in echocardiography. Data presented with \pm standard deviation

\begin{tabular}{|c|c|c|c|}
\hline Parameter & Feature & Group I & Group II \\
\hline \multirow{2}{*}{ PTX3 [ng/ml] } & Atherosclerosis in carotid arteries & $1.53 \pm 0.41$ & $1.05 \pm 0.95$ \\
\hline & No atherosclerosis in carotid arteries & $1.13 \pm 0.77$ & $1.26 \pm 0.55$ \\
\hline Significance & & $p=0.07$ & $p=0.12$ \\
\hline \multirow{2}{*}{ PTX3 [ng/ml] } & Normal EF & $1.18 \pm 0.42$ & $0.94 \pm 0.61$ \\
\hline & Impaired EF & $1.42 \pm 0.80$ & $1.66 \pm 1.39$ \\
\hline Significance & & $p=0.22$ & $p=0.08$ \\
\hline \multirow{2}{*}{ PTX3 [ng/ml] } & Correct mitral inflow E/A & $1.06 \pm 0.81$ & $0.91 \pm 0.63$ \\
\hline & Impaired left ventricle relaxation & $1.52 \pm 0.45$ & $1.38 \pm 1.09$ \\
\hline Significance & & $p=0.051$ & $p=0.061$ \\
\hline \multirow{2}{*}{ PTX3 [ng/ml] } & Correct mitral inflow $E^{\prime} / A^{\prime}$ & $0.82 \pm 0.71$ & $0.91 \pm 0.62$ \\
\hline & Impaired left ventricle relaxation & $1.6 \pm 0.42$ & $1.42 \pm 1.12$ \\
\hline Significance & & $p=0.058$ & $p=0.60$ \\
\hline
\end{tabular}

EF - ejection fraction 
Table 5. The correlation between PTX-3 and ultrasound measurements of carotid arteries

\begin{tabular}{lcc}
\hline & Group I & Group II \\
\hline CCA IMT av [mm] & $0.44^{*}$ & 0.19 \\
CCA IMT max [mm] & $0.42^{*}$ & 0.22 \\
ICA MV [cm/s] & 0.30 & 0.20 \\
ICA PI (pulsatility index) & 0.29 & 0.12 \\
ICA RI (resistance index) & 0.10 & 0.20
\end{tabular}

Data presented as $\mathrm{R}$ Spearman correlation. ${ }^{*} p<0.05$

CCA (IMT) av - common carotid artery (intima-media thickness) average; CCA (IMT) max - common carotid artery (intima-media thickness) maximum; ICA (MV) — internal carotid artery (mean velocity); ICA (PI) - internal carotid artery (pulsatility index); ICA (RI) - internal carotid artery (resistance index)

an association between plaque score and microvascular complications [23]. The association between duration of diabetes and presence of retinopathy seems obvious, but it is worth mentioning that just like retinopathy is also associated with age and chronic inflammation [24-26]. Therefore it is important to assess the relation between PTX3 concentration (an inflammatory biomarker) with the presence of retinopathy. In our study the concentration of PTX3 is lower in the group of patients with retinopathy than without it, but the difference is not significant, even after logarithmic transformation of the scores. Potentially, PTX3 seems to be associated with the presence of diabetic retinopathy but further cohort studies are necessary. Yang et al. described association of PTX3 with DR in a group of Korean patients with T2DM, where the mean PTX3 concentration was higher in a group of patients with retinopathy $(1.82 \pm 1.77 \mathrm{ng} / \mathrm{ml})[20]$.

In presented study PTX3 plasma concentration was associated with age of patients with diabetic retinopathy - the older the patient with retinopathy, the higher the PTX3 concentration. Moreover, in this group of patients the PTX3 concentration was higher among patients with higher $\mathrm{HbA}_{1 \mathrm{c}}$ value and longer duration of hypertension. It is surprising that all patients taking part in the study had very good diabetes control (mean value of $\mathrm{HbA}_{1 \mathrm{c}}=6.02 \pm 0.75$ among patients with retinopathy and $6.4 \pm 0.92$ among patient without retinopathy what can be cause by a small sample size and a single center study. Additionally we did not take into account hypoglycemia events rates and pharmacotherapy that could influence $\mathrm{HbA}_{1 \mathrm{c}}$ value.

So far, to our best knowledge, there were no such findings in the literature concerning patients with diabetic retinopathy. In some large population-based studies, there was an association found between the PTX3 higher concentration and age and the increased cardiovascular risk [27-29]. The correlation between
PTX3 concentration and diabetes control was found in two smaller studies but the outcome undoubtedly needs to be confirmed in larger studies [30, 31]. According to our study, there is a significant relation between PTX3, a marker of local inflammation and endothelial damage, and chronic hyperglicaemia, reflected by $\mathrm{HbA}_{1 \mathrm{c}}$ value but further and larger studies are necessary [32].

According to the literature, the measurement of intima-media complex can assess the progression of atherosclerosis and is an independent predictor of cardiovascular events [33]. The presence of plaque is defined as the intima-media thickness of the carotid artery above $1.5 \mathrm{~mm} \mathrm{[34].} \mathrm{We} \mathrm{have} \mathrm{proven} \mathrm{that}$ the mean and maximum intima-media thickness is significantly associated with PTX3 in the group of patients with diabetic retinopathy. The concentration of PTX3 increases with the increase of thickness of IMT of the carotid artery. The above correlation has an average statistical power, but on this basis it can be hypothethised that with the increase of PTX3 concentration, there is an increase of cardiovascular risk, as it was also proved in other population-based studies [27-29].

Atherosclerosis in carotid arteries was proven to be associated also with the presence of microvascular complications [23]. In presented study, the mean values of CCA ITM max in a group of patients with diabetic retinopathy were considerably below the cut-off value for the presence of atherosclerotic plaque $(1.5 \mathrm{~mm})$ and in spite of it, there was a significant correlation between it and PTX3 concentration. On this basis, there can be a conclusion drawn that PTX3 may be associated with the early stages of atherosclerosis in patients with diabetic retinopathy [21]. To our best knowledge, this is the first attempt to assess the association between PTX3 concentration and impaired systolic and diastolic cardiac function in a group of patients with diabetic retinopathy and it turned out that in this group of patients affected by left ventricle diastolic dysfunction there is a higher PTX3 plasma concentration comparing to patients without diabetic retinopathy, but the difference is not significant. The impaired left ventricle diastolic function is often symptomless and may lead to symptomatic heart failure in the future [35]. It is estimated that even if the diastolic dysfunction is present the risk of death in 3-5 years in 5 times greater than for patients with a proper diastolic function [36]. Similar conclusions on the PTX3 and impaired left ventricle diastolic function were drawn by Kimura et al. and Guo et al. but in a group of patients with normal glucose tolerance $[37,38]$. In another study Matsubara et al. propose that PTX3, which is produced in the coronary 
circulation by the cardiac muscle when the left ventricle diastolic function is worsening, is an independent marker of diastolic impairment and correlates with future cardiac events in patients with heart failure and normal ejection fraction $[39,40]$. The main limitation of presented study is the number of participants but the results encourage to further research.

\section{Conclusion}

According to the results obtained, there is no direct association between PTX3 concentration and the presence of diabetic retinopathy. On the other hand, there is an correlation between PTX3 and $\mathrm{HbA}_{1 c^{\prime}}$ age and IMT in carotid arteries in a group of type 2 diabetic patients with diabetic retinopathy found. Further studies in this field are neccessary.

\section{Conflict of interests}

The authors declare no conflicts of interests.

\section{REFERENCES}

1. Drakopoulou M, Toutouzas K, Michelongona A, et al. Vulnerable plaque and inflammation: potential clinical strategies. Curr Pharm Des. 2011; 17(37): 4190-4209, indexed in Pubmed: 22204378.

2. Pant S, Deshmukh A, Gurumurthy GS, et al. Inflammation and atherosclerosis - revisited. J Cardiovasc Pharmacol Ther. 2014; 19(2): 170-178, doi: 10.1177/1074248413504994, indexed in Pubmed: 24177335

3. Papatheodorou K, Banach M, Bekiari E, et al. Complications of Diabetes 2017. J Diabetes Res. 2018; 2018: 3086167, doi: 10.1155/2018/3086167, indexed in Pubmed: 29713648.

4. Resnikoff S, Pascolini D, Etya'ale D, et al. Global data on visual impairment in the year 2002. Bull World Health Organ. 2004; 82(11): 844-851, doi: /S0042-96862004001100009, indexed in Pubmed: 15640920.

5. Hendrick AM, Gibson MV, Kulshreshtha A. Diabetic Retinopathy. Prim Care. 2015; 42(3): 451-464, doi: 10.1016/j.pop.2015.05.005, indexed in Pubmed: 26319349.

6. Negi A, Vernon SA. An overview of the eye in diabetes. J R Soc Med. 2003; 96(6): 266-272, indexed in Pubmed: 12782689.

7. Defronzo RA. Banting Lecture. From the triumvirate to the ominous octet: a new paradigm for the treatment of type 2 diabetes mellitus. Diabetes. 2009; 58(4): 773-795, doi: 10.2337/db099028, indexed in Pubmed: 19336687.

8. Donath MY, Shoelson SE. Type 2 diabetes as an inflammatory disease. Nat Rev Immunol. 2011; 11(2): 98-107, doi: 10.1038/ /nri2925, indexed in Pubmed: 21233852

9. Mantovani A, Garlanda C, Bottazzi B, et al. The long pentraxin PTX3 in vascular pathology. Vascul Pharmacol. 2006; 45(5): 326-330, doi: 10.1016/j.vph.2006.08.011, indexed in Pubmed: 17023219.

10. International Diabetes Federation. IDF Diabetes Atlas, 8th edn. Brussels, Belgium: International Diabetes Federation, 2017. http:// www.diabetesatlas.org/.

11. Breviario F. d'Aniello EM. Golay J. et al. J Biol Chem. 1992; 267(31): 22190-22197. http://www.jbc.org/content/267/31/22190.

12. Szuszkiewicz-Garcia MM, Davidson JA. Cardiovascular disease in diabetes mellitus: risk factors and medical therapy. Endocrinol Metab Clin North Am. 2014; 43(1): 25-40, doi: 10.1016/j. ecl.2013.09.001, indexed in Pubmed: 24582090.
13. Gewurz $H$, Zhang $X H$, Lint TF. Structure and function of the pentraxins. Curr Opin Immunol. 1995; 7(1): 54-64, indexed in Pubmed: 7772283.

14. Lech M, Rommele C, Anders HJ. Pentraxins in nephrology: C-reactive protein, serum amyloid $\mathrm{P}$ and pentraxin-3. Nephrol Dial Transplant. 2013; 28(4): 803-811, doi: 10.1093/ndt/gfs448, indexed in Pubmed: 23243042.

15. Speeckaert MM, Speeckaert R, Carrero JJ, et al. Biology of human pentraxin 3 (PTX3) in acute and chronic kidney disease. J Clin Immunol. 2013; 33(5): 881-890, doi: 10.1007/s10875-013-9879-0, indexed in Pubmed: 23443958.

16. Ghosh P, Sahoo R, Vaidya A, et al. Role of complement and complement regulatory proteins in the complications of diabetes. Endocr Rev. 2015; 36(3): 272-288, doi: 10.1210/er.2014-1099, indexed in Pubmed: 25859860.

17. Iwasaki A, Medzhitov R, Iwasaki A, et al. Regulation of adaptive immunity by the innate immune system. Science. 2010; 327(5963): 291-295, doi: 10.1126/science.1183021, indexed in Pubmed: 20075244.

18. Bottazzi B, Garlanda C, Salvatori G, et al. Pentraxins as a key component of innate immunity. Curr Opin Immunol. 2006; 18(1): 10-15, doi: 10.1016/j.coi.2005.11.009, indexed in Pubmed: 16343883.

19. Zhou W, Hu W. Serum and Vitreous Pentraxin 3 Concentrations in Patients with Diabetic Retinopathy. Genet Test Mol Biomarkers. 2016; 20(3): 149-153, doi: 10.1089/gtmb.2015.0238, indexed in Pubmed: 26836044.

20. Yang HS, Woo JE, Lee SJ, et al. Elevated plasma pentraxin 3 levels are associated with development and progression of diabetic retinopathy in Korean patients with type 2 diabetes mellitus. Invest Ophthalmol Vis Sci. 2014; 55(9): 5989-5997, doi: 10.1167/ /iovs.14-14864, indexed in Pubmed: 25159210.

21. Nabrdalik K, Chodkowski A, Bartman W, et al. Pentraxin 3 and atherosclerosis among type 2 diabetic patients. Open Life Sciences. 2017; 12(1): 92-98, doi: 10.1515/biol-2017-0010.

22. Lee R, Wong TY, Sabanayagam C. Epidemiology of diabetic retinopathy, diabetic macular edema and related vision loss. Eye Vis (Lond). 2015; 2: 17, doi: 10.1186/s40662-015-0026-2, indexed in Pubmed: 26605370.

23. Bartman W, Nabrdalik K, Kwiendacz H, et al. Association between carotid plaque score and microvascular complications of type 2 diabetes. Pol Arch Intern Med. 2017; 127(6): 418-422, doi: 10.20452/pamw.4024, indexed in Pubmed: 28508856

24. Massengill MT, Ahmed CM, Lewin AS, et al. Neuroinflammation in Retinitis Pigmentosa, Diabetic Retinopathy, and Age-Related Macular Degeneration: A Minireview. Adv Exp Med Biol. 2018; 1074: 185-191, doi: 10.1007/978-3-319-75402-4 23, indexed in Pubmed: 29721943.

25. Gökşen D, Levent $E$, Kar $S$, et al. Serum adiponectin and hsCRP levels and non-invasive radiological methods in the early diagnosis of cardiovascular system complications in children and adolescents with type 1 diabetes mellitus. J Clin Res Pediatr Endocrinol. 2013; 5(3): 174-181, doi: 10.4274/Jcrpe.1003, indexed in Pubmed: 24072086.

26. Yu HI, Sheu WHH, Song YM, et al. C-reactive protein and risk factors for peripheral vascular disease in subjects with Type 2 diabetes mellitus. Diabet Med. 2004; 21(4): 336-341, doi: 10.1111/j.14645491.2004.01144.x indexed in Pubmed: 15049935.

27. Jenny NS, Arnold AM, Kuller LH, et al. Associations of pentraxin3 with cardiovascular disease and all-cause death: the Cardiovascular Health Study. Arterioscler Thromb Vasc Biol. 2009; 29(4): 594-599, doi: 10.1161/ATVBAHA.108.178947, indexed in Pubmed: 19164811.

28. Jylhävä J, Haarala A, Kähönen M, et al. Pentraxin 3 (PTX3) is associated with cardiovascular risk factors: the Health 2000 Survey. Clin Exp Immunol. 2011; 164(2): 211-217, doi: 10.1111/j.13652249.2011.04354.x indexed in Pubmed: 21391986.

29. Jenny NS, Blumenthal RS, Kronmal RA, et al. Associations of pentraxin 3 with cardiovascular disease: the Multi-Ethnic Study 
of Atherosclerosis. J Thromb Haemost. 2014; 12(6): 999-1005, doi: 10.1111/jth.12557, indexed in Pubmed: 24628740.

30. Vilahur G, Badimon L. Biological actions of pentraxins. Vascul Pharmacol. 2015; 73: 38-44, doi: 10.1016/j.vph.2015.05.001, indexed in Pubmed: 25962566.

31. Napoleone E. Long Pentraxin PTX3 Upregulates Tissue Factor Expression in Human Endothelial Cells: A Novel Link Between Vascular Inflammation and Clotting Activation. Arteriosclerosis, Thrombosis, and Vascular Biology. 2002; 22(5): 782-787, doi: 10.1161/01.atv.0000012282.39306.64.

32. Koga S, Ikeda S, Yoshida T, et al. Elevated levels of systemic pentraxin 3 are associated with thin-cap fibroatheroma in coronary culprit lesions: assessment by optical coherence tomography and intravascular ultrasound. JACC Cardiovasc Interv. 2013; 6(9): 945-954, doi: 10.1016/j.jcin.2013.04.024, indexed in Pubmed: 23954061.

33. Polak JF, Pencina MJ, Pencina KM, et al. Carotid-wall intima-media thickness and cardiovascular events. N Engl J Med. 2011; 365(3): 213-221, doi: 10.1056/NEJMoa1012592, indexed in Pubmed: 21774709.

34. Polak JF, Szklo M, Kronmal RA, et al. The value of carotid artery plaque and intima-media thickness for incident cardiovascular disease: the multi-ethnic study of atherosclerosis. J Am Heart Assoc. 2013; 2(2): e000087, doi: 10.1161/JAHA.113.000087, indexed in Pubmed: 23568342.

35. Peri G, Introna M, Corradi D, et al. PTX3, A prototypical long pentraxin, is an early indicator of acute myocardial infarction in humans. Circulation. 2000; 102(6): 636-641, indexed in Pubmed: 10931803.

36. Latini R, Maggioni AP, Peri G, et al. Lipid Assessment Trial Italian Network (LATIN) Investigators. Prognostic significance of the long pentraxin PTX3 in acute myocardial infarction. Circulation. 2004; 110(16): 2349-2354, doi: 10.1161/01.CIR.0000145167.30987.2E, indexed in Pubmed: 15477419.

37. Kimura S, Inagaki H, Haraguchi Go, et al. Relationships of Elevated Systemic Pentraxin-3 Levels With High-Risk Coronary Plaque Components and Impaired Myocardial Perfusion After Percutaneous Coronary Intervention in Patients With ST-Elevation Acute Myocardial Infarction. Circulation Journal. 2014: 159-169, doi: 10.1253/circj.cj-13-0329.

38. Guo R, Li Y, Wen J, et al. Elevated plasma level of pentraxin-3 predicts in-hospital and 30-day clinical outcomes in patients with non-ST-segment elevation myocardial infarction who have undergone percutaneous coronary intervention. Cardiology. 2014; 129(3): 178-188, doi: 10.1159/000364996, indexed in Pubmed: 25323314.

39. Matsubara J, Sugiyama S, Nozaki T, et al. Incremental prognostic significance of the elevated levels of pentraxin 3 in patients with heart failure with normal left ventricular ejection fraction. J Am Heart Assoc. 2014; 3(4), doi: 10.1161/JAHA.114.000928, indexed in Pubmed: 25012287.

40. Kaess BM, Vasan RS. Heart failure: pentraxin 3 - a marker of diastolic dysfunction and HF? Nat Rev Cardiol. 2011; 8(5): 246-248, doi: 10.1038/nrcardio.2011.50, indexed in Pubmed: 21448138. 